\title{
Coherent regimes of mutually coupled Chua's circuits
}

\author{
I. Gomes Da Silva* \\ Instituto Mediterráneo de Estudios Avanzados IMEDEA (CSIC-UIB), Campus Universitat Illes Balears, \\ E-07122 Palma de Mallorca, Spain \\ S. De Monte ${ }^{\dagger}$ \\ Department of Biology, UMR 7625, Ecole Normale Supérieure, Paris, France \\ F. d'Ovidio \\ Laboratoire de Météorologie Dynamique, UMR 8539, Ecole Normale Supérieure, Paris, France \\ R. Toral ${ }^{\S}$ \\ Instituto Mediterráneo de Estudios Avanzados IMEDEA (CSIC-UIB), Campus Universitat Illes Balears, \\ E-07122 Palma de Mallorca, Spain \\ C. R. Mirasso" \\ Departament de Física, Universitat de les Illes Baleares, Campus Universitat Illes Balears, E-07122 Palma de Mallorca, Spain
}

(Received 25 August 2005; published 2 March 2006)

\begin{abstract}
We study the dynamical regimes that emerge from the strong coupling between two Chua's circuits with parameters mismatch. For the region around the perfect synchronous state we show how to combine parameter diversity and coupling in order to robustly and precisely target a desired regime. This target process allows us to obtain regimes that may lie outside parameter ranges accessible for any isolated circuit. The results are obtained by following a recently developed theoretical technique, the order parameter expansion, and are verified both by numerical simulations and on electronic circuits. The theoretical results indicate that the same predictable change in the collective dynamics can be obtained for large populations of strongly coupled circuits with parameter mismatches.

DOI: 10.1103/PhysRevE.73.036203

PACS number(s): 05.45.Gg, 07.50.Ek
\end{abstract}

\section{INTRODUCTION}

One of the most important features of interacting oscillating units is the possibility for their motions to entrain each other and evolve in synchronicity. The phenomenon of synchronization is a universal feature underlying the collective behavior of populations of dynamical systems and its importance has been recognized in many different fields [1-3]. In particular, a great deal of attention has been devoted to globally coupled dynamical systems as a model for a variety of physical [4], chemical [5,6], and biological systems [7,8].

Even if several theoretical approaches assume that populations are composed of identical units, real systems display an unavoidable diversity among the individual oscillators, e.g., a mismatch in their parameters. The effect of such a diversity might hinder synchronization and yields an incoherent regime if the coupling is too weak. The main feature of incoherence is that the motions of the individual systems are largely independent of each others. The collective dynamics, then, is either stationary on average (for an infinite

\footnotetext{
*Electronic address: iacyel@imedea.uib.es

†Electronic address: demonte@biologie.ens.fr

‡Electronic address: dovidio@1md.ens.fr

${ }^{\S}$ Electronic address: raul@imedea.uib.es

"Electronic address: claudio@galiota.uib.es
}

number of elements) or displays fluctuations that scale with the population size. When the coupling strength is increased, some or all of the population elements synchronize and, as a result, oscillations start to be detected at a macroscopic level. The transition to collective oscillations is typically accompanied by rather complex regimes, such as clustering [9], partial synchronization [10], phase synchronization [11], etc. For even stronger coupling, complete synchronous regimes, characterized by a high degree of coherency within the population, develops. This means that all the population elements share the same kind of dynamics, apart from small differences due to the microscopic diversity, and this dynamics reflects at the macroscopic level.

Despite the numerous theoretical advances in the analysis of globally coupled dynamical systems, the experimental verification has been revealed to be extremely difficult. The Kuramoto transition from incoherence to the locked state is in this sense a good example [12]. It dates back to 1975 and is one of the best known and general results in synchronization theory. However, to our best knowledge, it has been quantitatively verified only once in experiments and required the implementation of an ad hoc system [13]. Two main problems are encountered in experiments. One is the difficulty in controlling or measuring the parameter variability in the system. The second one is related to the global coupling, that occurs seldom in nature and is instead a limit case of local coupling with large correlation length. These experimental limitations are especially relevant for regimes close 
to incoherence, that is very fragile with respect to changes in the microscopic properties, as well as to finite-size effects.

In order to avoid these problems associated with incoherence, in this paper we focus instead on globally coupled systems close to the synchronous state. As was recently shown, such coherent states do not strongly depend on the population size $[14,15]$. This fact is very interesting from the experimental viewpoint, since the collective behavior can be studied in systems of only two elements and the results extended to larger populations. Moreover, the coherent dynamics can be described by a set of low-dimensional equations with only few parameters. Transitions among different regimes correspond to bifurcations involving a small number of degrees of freedom and parameters, thus having the advantage of being very robust to changes in the microscopic features of the population.

As an application of such theoretical results, we study the strong coupling regimes of two Chua's circuits. Chua's circuits offer an intermediate step between theory and applications. They can be modeled with a simple set of nonlinear equations, thus allowing the use of analytical methods and quick numerical simulations. At the same time they can be easily implemented in hardware. Even if they constitute a rather controlled experimental system, a verification of theoretical results faces a test on its robustness with respect to (i) noise fluctuations in states and parameter values, (ii) constraints in the accessible parameter precision and ranges, and (iii) small functional mismatches between model and implementation.

Section II derives the equations for the macroscopic dynamics of an interacting population (of any size) in the coherent regime. This representation allows us to infer on the dynamical regimes of the experimental system. We then provide two examples of comparison between theoretical prediction and experimental results. In Sec. III we show how the parameter mismatch can be used to drive both the mean field and the individual circuits to regimes that are different from the uncoupled dynamics. In Sec. IV we consider the case in which the individual elements differ for the time scale of their motion, and focus in particular on the regime of oscillator death, where parameter diversity induces a suppression of the individual and collective oscillations. Finally, Sec. V is devoted to the discussion of the results and to their possible applications.

\section{ORDER PARAMETER EXPANSION OF MUTUALLY COUPLED CHUA'S CIRCUITS}

The order parameter expansion [14-16] is a useful technique for studying regimes that bifurcate from the perfectly synchronous state in populations of globally and strongly coupled elements when the parameter mismatch is increased. This approach also describes the changes in coherent behavior that take place in diverse populations when the coupling is modified. Let us here review the main results by considering a set of equations of the form

$$
\dot{x}_{j}=f\left(x_{j}, p_{j}\right)+K\left(X-x_{j}\right), \quad j=1,2, \ldots, N,
$$

where $p_{j}$ is a parameter different in every individual system and the dynamics of each element $f_{j}\left(x_{j}\right)=f\left(x_{j}, p_{j}\right)$, is defined by the smooth function $f_{j}: \mathbb{R}^{n} \rightarrow \mathbb{R}^{n}$. All the elements are coupled to the the mean field of the population $X=\left\langle x_{j}\right\rangle$ through the coupling function $K\left(X-x_{j}\right)$ that, as we will see later, for the case of the electronic coupling we use, can be taken as a linear diagonal operator (in fact, a more general system can be considered, see Refs. $[15,16]$ for details.) The basic idea of the method is to obtain an effective equation of motion for the mean field variable $X$, valid when all the elements evolve in time close to $X$. This is done by considering regimes that are perturbations of the perfectly synchronous state, defined as $x_{j}=X, \forall j$. Under this assumption, Eq. (1) can be expanded into the individual deviations from the mean field $\epsilon_{j}=x_{j}-X$ and from the mean parameter $\delta_{j}=p_{j}$ $-p_{0}$, where $p_{0}=\langle p\rangle$. By averaging Eq. (1) over the population, one gets, at first order, the following approximated equation for the mean field:

$$
\begin{gathered}
\dot{X}=F\left(X, p_{0}\right)+D_{x, p} F\left(X, p_{0}\right) W \\
\dot{W}=\sigma^{2} D_{p} F\left(X, p_{0}\right)+\left[D_{x} F\left(X, p_{0}\right)-K\right] W,
\end{gathered}
$$

where $W=\langle\delta \epsilon\rangle$ is a second macroscopic variable or shape order parameter, $p_{0}$ is the average parameter value, and $\sigma^{2}$ is the standard deviation of the parameter distribution. The derivative operators are defined as follows: $D_{x}=\partial / \partial x, D_{p}$ $=\partial / \partial p$, and $D_{x, p}=\partial^{2} / \partial x \partial p$. In addition to being low dimensional, such equation has the advantage of having a clear physical meaning: the mean field behaves similar to the average element, perturbed by the macroscopic variable $W$ $=\langle\delta \epsilon\rangle$ that quantifies the mismatch in phase and parameter space. The macroscopic regimes appear as an unfolding of the perfectly locked state $\dot{X}=F\left(X, p_{0}\right)$ and are controlled by the parameter $K$ and $\sigma^{2}$. Such equation holds when the displacements from the mean field $\epsilon_{j}$ are small, and thus, operationally, when the coupling is sufficiently strong compared with the parameter mismatch. Another important consideration is that Eq. (2) does not depend on the population size. Consequently, the results we obtain for two Chua's circuits can be readily generalized to larger arrays.

Let us now apply Eq. (2) to the case of two mutually coupled Chua's circuits. The configuration of the circuits that we study is shown in Fig. 1. The equations are easily written in terms of the voltages $v^{1}$ and $v^{2}$ (at point 1 and 2 , respectively) and the current $I$ across the inductance (point 3 ), the first circuit is described by

$$
\begin{aligned}
\dot{v}_{1}^{1}= & \frac{1}{C_{1}^{1} R_{1}} v_{1}^{2}-\frac{G_{1}^{a}-G_{1}^{b}}{2 C_{1}^{1}}\left(\left|v_{1}^{1}+B_{1}^{p}\right|-\left|v_{1}^{1}-B_{1}^{p}\right|\right) \\
& -\frac{1}{C_{1}^{1}}\left(G_{1}^{b}+\frac{1}{R_{1}}\right) v_{1}^{1}+\frac{1}{C_{1}^{1} R_{1}^{k}}\left(v_{1}^{1}-v_{2}^{1}\right), \\
\dot{v}_{1}^{2}= & \frac{1}{C_{1}^{2}} I^{1}+\frac{1}{C_{1}^{2} R_{1}}\left(v_{1}^{1}-v_{1}^{2}\right)+\frac{1}{C_{1}^{2} R_{2}^{k}}\left(v_{1}^{2}-v_{2}^{2}\right),
\end{aligned}
$$




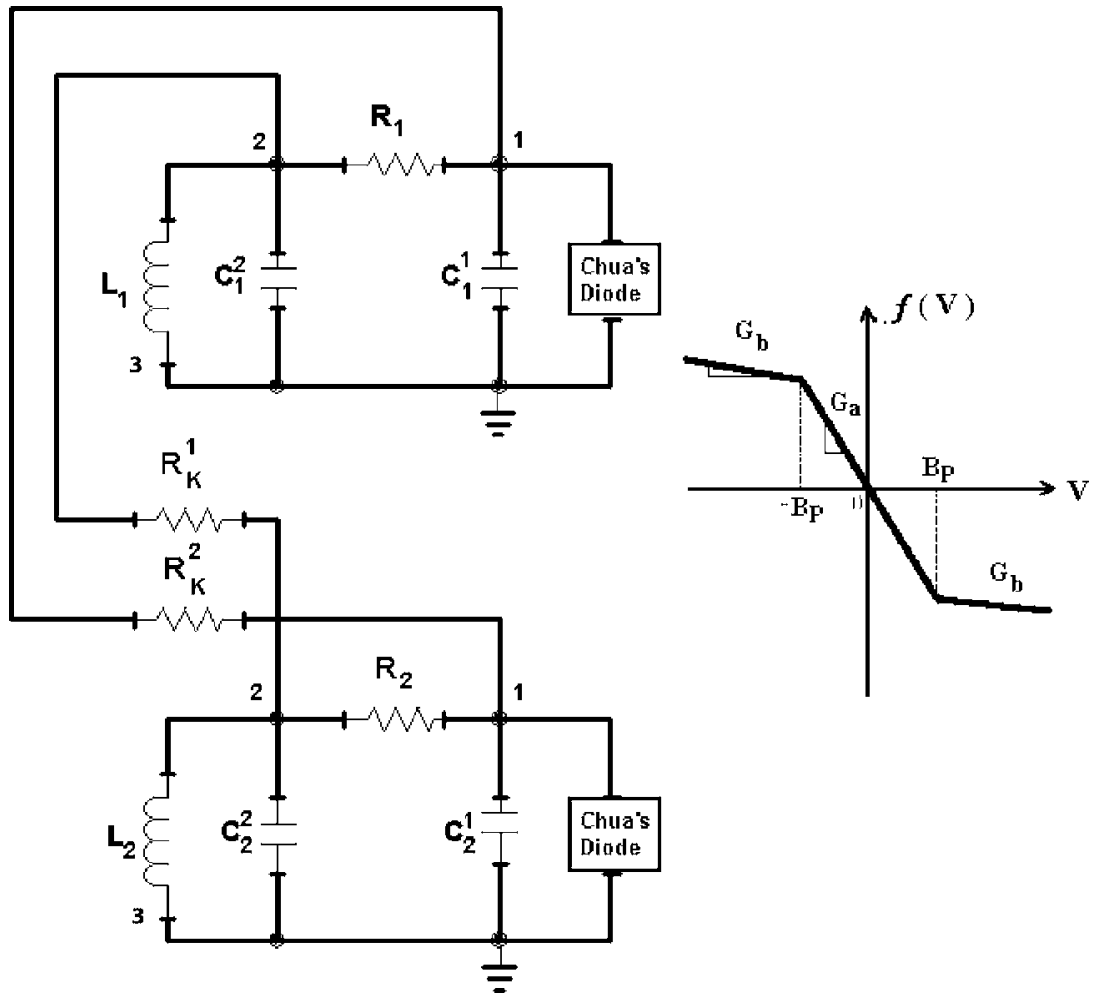

FIG. 1. Scheme of the two mutually coupled Chua's circuits. The parameters are $C_{1,2}^{1}=10.2 \mathrm{nF}$, $C_{1,2}^{2}=101.8 \mathrm{nF}, \quad L_{1,2}=18.4 \mathrm{mH}$, $G_{1,2}^{a}=-7.625 \times 10^{-4}, \quad$ and $G_{1,2}^{b}$ $=4.12 \times 10^{-4}$.

$$
\dot{I}_{1}=-\frac{r_{1}^{b}}{L_{1}} I_{1}-\frac{1}{L_{1}} v_{1}^{2},
$$

where $R_{k}^{1,2}$ are the two coupling resistors. These resistors will be used as bifurcation parameters. The other internal parameters are displayed in the caption of Fig. 1. Unless explicitly stated otherwise, these are the values that will be used in the next sections. The second circuit has an equivalent equation, with lower index 2 instead. Before proceeding with the analysis, it is worth mentioning that the mutual coupling Eq. (3) term can be recast in the form of a global coupling term, so that the correspondence with Eq. (2) is readily established. The dependence on the mean field becomes apparent by writing

$$
\left(v_{2}^{1}-v_{1}^{1}\right)=\left(v_{2}^{1}+v_{1}^{1}-2 v_{1}^{1}\right)=2\left(\left\langle v_{1}\right\rangle-v_{1}^{1}\right) .
$$

The coupling constants are the multiplicative factors $1 /\left(C^{1} R_{k}^{1}\right)$ and $1 /\left(C^{2} R_{k}^{2}\right)$. At this point, we recover Eq. (1) by identifying $x_{j}$ with the vector $\left\langle v_{j}^{1}, v_{j}^{2}, I_{j}\right\rangle$ and $f_{j}\left(x_{j}\right)$ with the equation for one uncoupled Chua circuit. The coupling matrix is

$$
K^{1}=\left[\begin{array}{ccc}
\frac{1}{C_{1}^{1} R_{k}^{1}} & 0 & 0 \\
0 & \frac{1}{C_{1}^{2} R_{k}^{2}} & 0 \\
0 & 0 & 0
\end{array}\right] .
$$

In the case when the two circuits have identical capacities, the coupling is symmetrical (a more relaxed assumption will be presented in Sec. IV). The explicit calculation of the reduced equations are given in the Appendix. With this formal- ism, we are now ready to use Eq. (2) on some specific condition and to study the collective regimes that emerge due to the interplay of parameter mismatch and coupling.

\section{STRONG MUTUAL COUPLING}

Let us first address the case in which the mismatch between the two circuits is limited to the value of the internal resistors $R_{1,2}$, while the other internal parameters are kept identical. The coupling resistors $R_{k}^{1,2}$ are chosen weak enough to ensure that the coupling is strong and the regime coherent. Under such strong coupling conditions, the collective dynamics will be qualitatively equal to the dynamics of an "average oscillator." This corresponds to a system of equations identical to those of each single element, except for the parameter on which diversity is imposed, that is instead substituted by its average value. Intuitively, such property of the macroscopic dynamics is due to the fact that, when the coupling matrix is predominantly diagonal, the shape parameter $W$ remains small. This corresponds to the situation in which the population is tightly packed around the mean field. In the limit in which the coupling is infinitely strong, $W$ vanishes and in Eq. (2) we end up with the equation for an average oscillator.

Such a consideration has interesting implications in the control of the collective behavior of populations of dynamical systems, as well as in the detection of the microscopic features in terms of purely macroscopic observations. Indeed, the dynamics that is stabilized by coupling nonidentical circuits may not be attainable by any of them if uncoupled. The collective regime is thus "coded" in the diversity and coupling rather than in the internal features of the individual circuits. Moreover, Eq. (2) allows a straight- 

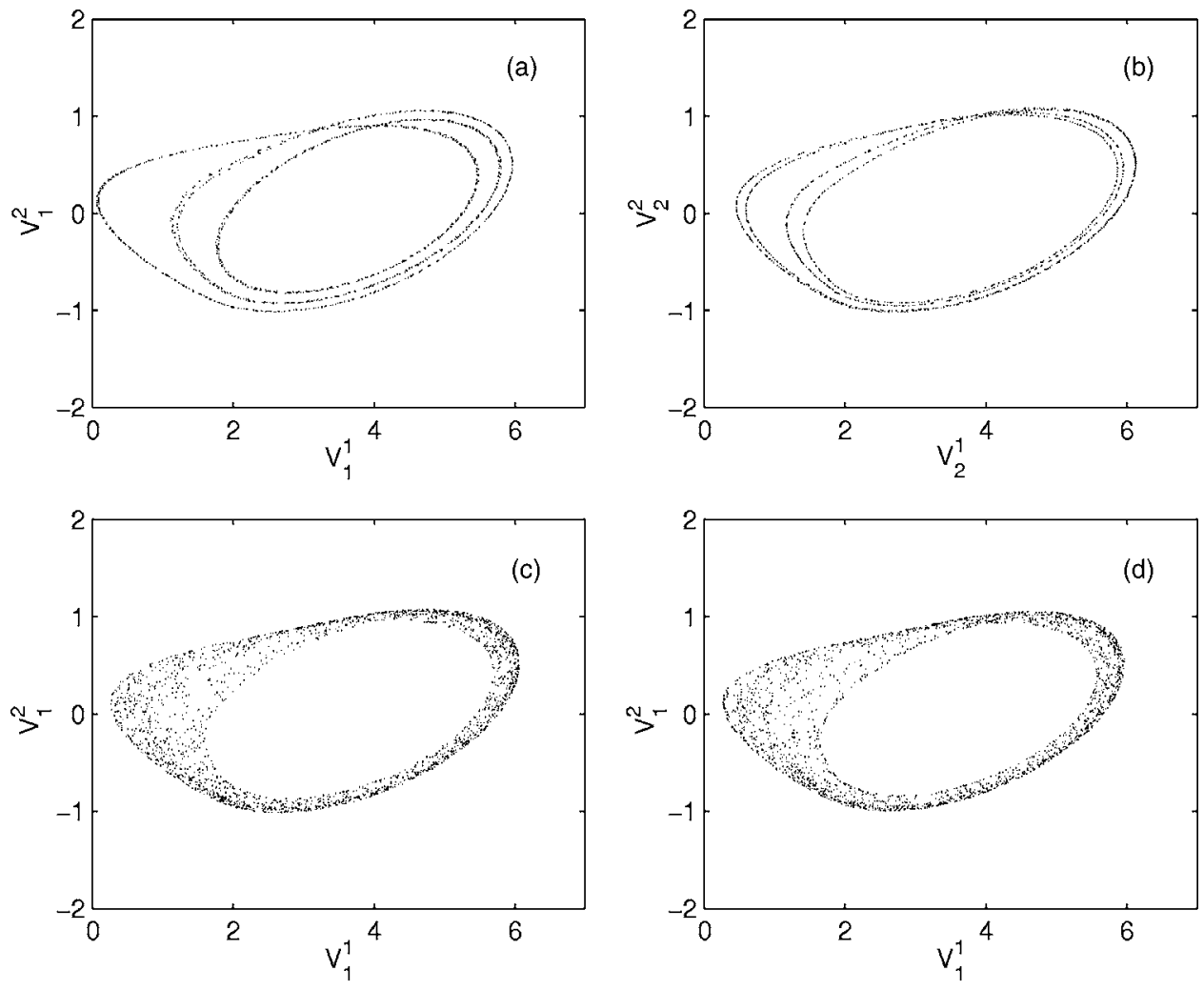

forward identification of such a hidden collective behavior.

As a first application of the aforementioned theoretical results, let us examine the case when both circuits operate in a periodic regime. Figures 2(a) and 2(b) display the experimentally measured attractors of the two uncoupled circuits, of periods 3 and 4, respectively. Figure 2(c) shows that, as a result of the coupling, both oscillators behave chaotically, although the mismatch in the parameters has only minor effects on their individual dynamics. As predicted by Eq. (2), the regime obtained by strongly coupling the circuits is the same one observed in an isolated circuit with the internal resistor set to the averaged value [Fig. 2(d)].

The same idea can be used for stabilizing unstable periodic orbits. Let us, for instance, consider the case in which both circuits are chaotic, but their parameter values are located, when the circuits are uncoupled, on the opposite side of a periodic window. Figure 3 shows that once they are coupled, both circuits get synchronized onto a period-three orbit. The coupling thus results into the stabilization of an unstable orbit embedded into the chaotic attractor.

The possibility of obtaining a qualitatively novel dynamics by coupling two circuits with different internal parameters is robust with respect to changes in the coupling intensity (also under differential modifications of the resistors) as long as both coupling resistors are sufficiently weak, that is, coupling is sufficiently strong. This robustness was confirmed by repeating the two experiments described above for several values of the coupling resistors.

In addition to providing a way for driving the system to specific regimes, the experiments also show that the mathematical limit of infinitely strong coupling is actually attainable, to a good approximation, even for experimentally feasible resistance values. It is also important to remark that in order to target a regime one only needs to know the bifurcation diagram of an isolated circuit: this information can be obtained experimentally and in principle does not require the knowledge of the functional form of $f$.

\section{OSCILLATION DEATH IN CIRCUITS WITH TIME SCALE MISMATCH}

In this section we address changes in the collective regimes due to modifications in the coupling intensity. In particular, we will provide an experimental realization of oscillator death for coupled chaotic systems. In this regime the dynamics of each population element is suppressed as a collective effect, i.e., resulting from the interplay of coupling and diversity. Oscillator death has been first described in populations of limit cycle oscillators with direct $[17,18]$ or time-delayed coupling [19]. Recently, an order parameter expansion has been used to demonstrate that this regime may arise under generic conditions in populations of globally coupled element with time scale mismatch [15]. The equations for a population with time scale mismatch and global, linear coupling can be written as

$$
\dot{x}_{j}=\tau_{j} f\left(x_{j}\right)+K\left(X-x_{j}\right), \quad j=1,2, \ldots, N .
$$

This equation may be seen as a simple way for generalizing the Kuramoto model, where the distributed parameter is the natural frequency of the oscillators, to an individual dynamics different from a limit cycle. The peculiar parameter de- 

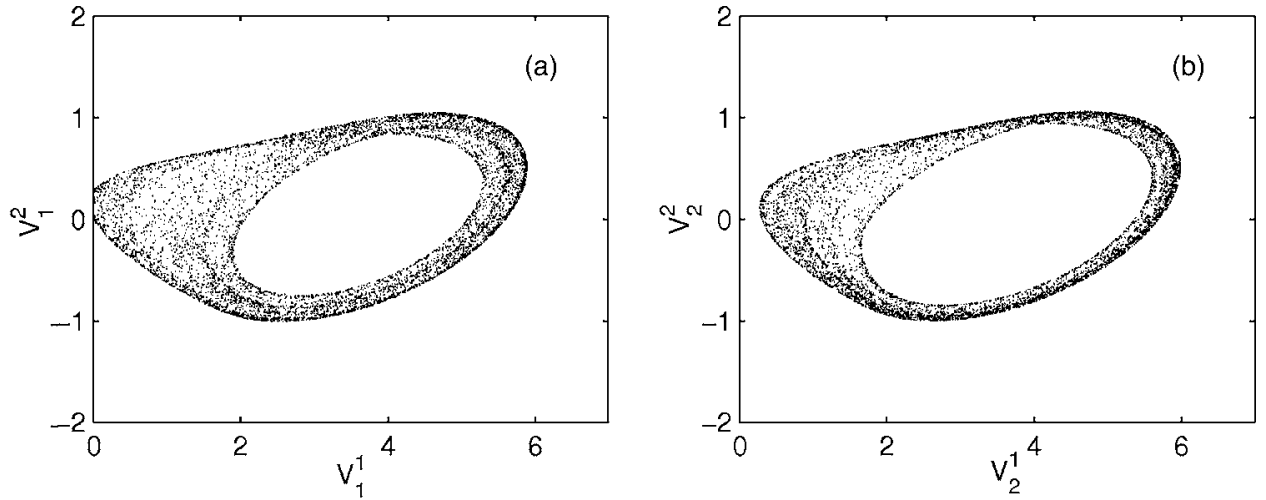

FIG. 3. Stabilization of an unstable period-three orbit by coupling of two nonidentical chaotic circuits. (a) and (b) Uncoupled chaotic dynamics of the two circuits $\left(R_{1}=1621, R_{2}=1745 \mathrm{Ohm}\right)$. (c) Attractor of the first circuit after the coupling $\left(R_{k}^{1,2}=200 \mathrm{Ohm}\right)$ is established. As for Fig. 2, the
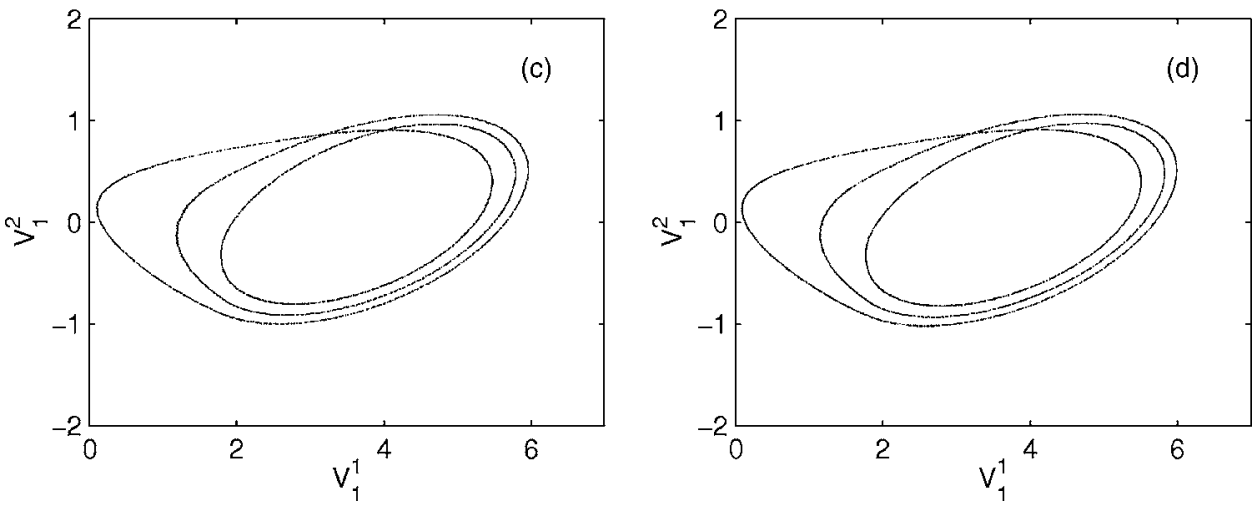
two circuit are strongly synchronized and thus only one attractor is shown. (d) The dynamics of the coupled circuits can be predicted by a single circuit with the internal resistance at the average value $\left(R_{1}+R_{2}\right) / 2=1683 \mathrm{Ohm}$.

pendence of Eq. (6) is reflected in a simple form of the reduced system

$$
\begin{gathered}
\dot{X}=\langle\tau\rangle f(X)+J W, \\
\dot{W}=\sigma^{2} f(X)+(\langle\tau\rangle J-K) W,
\end{gathered}
$$

where $J$ is the Jacobian $D_{x} f(x)$.

Due to the multiplicative nature of the distributed parameter in Eq. (6), any fixed point of $f$ is also a fixed point for the mean field. The stability of such a point, however, can change due to the presence of the coupling and time scale mismatch. With some algebra one finds that generic conditions exist under which an unstable focus of $f$ becomes attracting for the population. Referring to Ref. [15] for the details, here we just notice that, if the time scale mismatch is sufficiently wide, this equilibrium is stabilized for "intermediate" values (i.e., large enough to avoid incoherence and smaller than those where nonstationary coherent solutions are present) of the coupling.

For the case of Chua circuits, one can see from Eq. (3) that the time scale can be easily changed by tuning the capacitors $C_{1}, C_{2}$, and the inductance $L$. We modify the time scale of one of the two circuits by making use of commercially available capacitors and inductances, thus obtaining a timescale mismatch of $14 \%$. Both circuits, when uncoupled, have similar chaotic dynamics (double scroll attractor) although their time evolution takes place with different speed.

The bifurcation diagram of the reduced system Eq. (7) can be numerically computed for an interval of coupling intensities comparable to that accessible in our experimental setup. The diagram provides the coherent regimes of diffusively coupled Chua circuits with time scale mismatch as a function of the coupling strength. The standard deviation $\sigma$ of the time scale distribution is set to 0.14 . As expected from the previous section, for a low value of the coupling resistors (strong coupling), the circuits are entrained on a chaotic attractor indistinguishable from the uncoupled dynamics. However, when the coupling is reduced a cascade of coherent regimes bifurcates from the chaotic attractor and eventually the state of oscillator death is reached (Fig. 4). Such diagram is independent of the population as far as the coupling is global and diffusive [15].

The resistances $R_{k}^{1}=R_{k}^{2}=R_{k}$ of the coupled circuits can now be tuned in order to target a specific collective regime, according to the numerical characterization of the reduced system. Figure 5 shows some of the emergent dynamics experimentally observed. The oscillation death regime appears in the circuits for $R^{k}$ slightly above $1.5 \mathrm{k} \Omega$ (not shown).

\section{CONCLUSIONS}

Although the importance of emergent behavior in populations of nonidentical dynamical systems is widely recognized, only a few experimental systems offer the necessary degree of control for testing the theoretical results. Here, we have proposed the implementation of a circuit aimed at mimicking the coherent behavior of populations with parameter mismatch in the region of strong coupling. Theoretical results and numerical simulations suggest that this case is representative of larger populations and constitute a tool for addressing diversity-dependent coherent dynamics.

The experimental system we have used is composed by two symmetrically coupled Chua circuits. The diversity in 


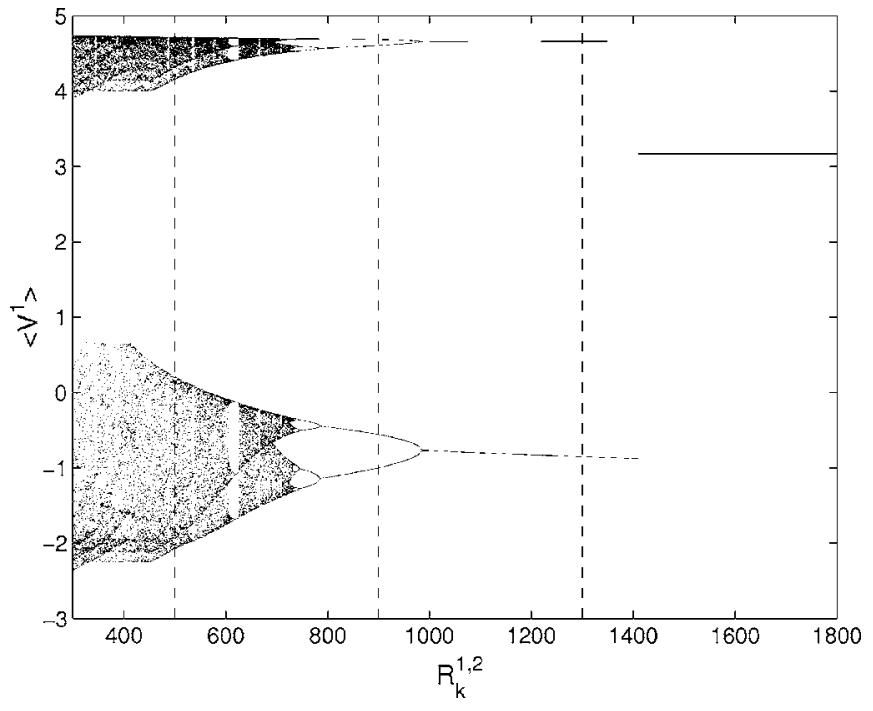

FIG. 4. Bifurcation diagram of the reduced system Eq. (7). The Poincaré section of the attractor is shown as a function of the coupling strength for a population of circuits with identical internal resistor set at $1562 \mathrm{Ohm}$, a time scale distribution of 0.14 and diffusive, global coupling. By tuning the coupling resistor the system undergoes a transition from a two-lobe to a one-lobe attractor. This macroscopic attractor then undergoes a backward period-doubling bifurcation cascade to a limit cycle, and eventually a fixed point is stabilized through a subcritical bifurcation.

their components introduces a mismatch in the internal parameters. We have shown that the average dynamics of such coupled systems can be very different from the one observed in the individual circuits when decoupled. The order parameter expansion developed for populations of strongly coupled dynamical systems allowed us to predict such a collective behavior based on the knowledge of the uncoupled dynamics. By controlling the parameter mismatch, we can target different dynamical regimes. For the Chua's circuits, such collective regimes can also be obtained by tuning the internal resistor of an uncoupled circuit, but this is not at all a requirement. On other systems the variability of uncoupled regimes may be much more limited. For these cases, the interplay of strong coupling and parameter mismatch can be a valuable technique for extending the dynamics outside the region of the uncoupled regimes. The possibility of robustly targeting a specific coherent regime can find a wide applicability in populations of globally coupled nonidentical systems, the chaotic behavior of Chua circuits being just one example of individual dynamics. As a second example of diversity-induced qualitative changes of the collective behavior, we have addressed the case in which the circuits differ by their time scales and studied the oscillation death scenario.

The coherency of the dynamics is not only the basic assumption that allows us to analytically address the collective regimes, but also guarantees that the results obtained for two circuits can be extended to populations of many elements with a comparable variance of the parameter diversity. The robustness of strong coupling regimes to changes in the number of interacting circuits is confirmed by numerical simulations. This shows indeed that the collective behavior is selected according to the variance rather than from the actual shape of the parameter distribution or the population size.

\section{ACKNOWLEDGMENTS}

S.D.M. is thankful for the hospitality of DFI-IMEDEA and was supported by the EIF Grant No. 010169. The au-
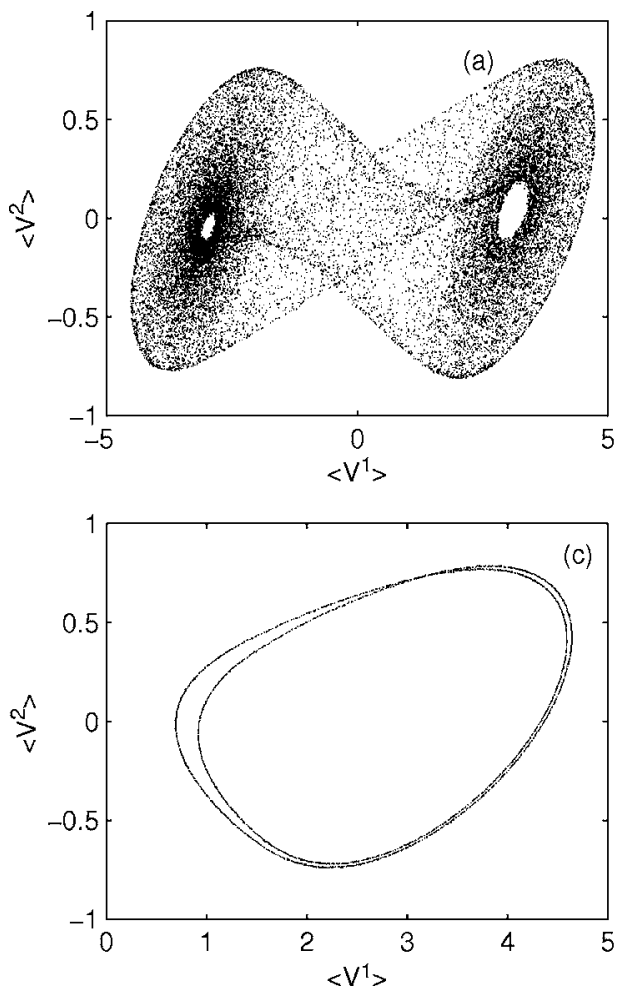
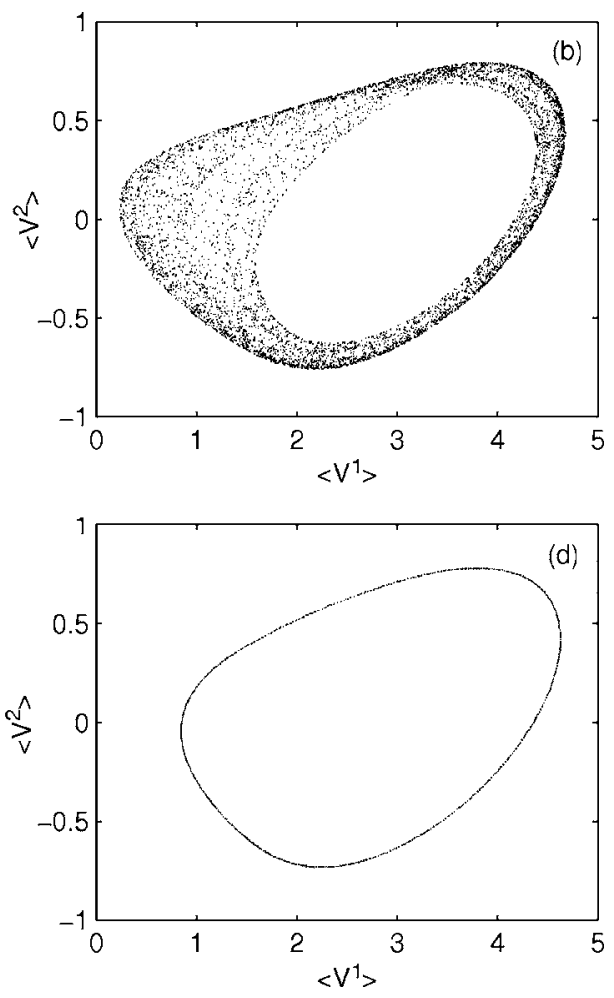

FIG. 5. Experimental behavior of the coupled circuits at (a) maximum coupling $R_{k}^{1,2}=0$; (b) $-(\mathrm{d})$ three decreasing coupling intensities, corresponding to the resistor values $R_{k}^{1,2}=500, \quad 900, \quad$ and $1300 \mathrm{Ohm}$. The Poicaré section of the reduced system Eq. (7) for these cases are indicated by the vertical dashed lines superimposed to the bifurcation diagram of Fig. 4. 
thors thank W. Korneta for interesting discussions and M. Oprandi for technical help. This work has been partly supported by MEC (Spain) and FEDER through Project CONOCE2 (Grant No. FIS2004-00953). The authors acknowledge support from the projects FIS2004-5073C04-03 and BIOSIM (EU NoE Grant LSHB-CT-2004005137).

\section{APPENDIX}

The equations approximating the average dynamics of a population with time scale mismatch can be computed for the Chua circuits that we have addressed in this paper starting from Eq. (3). The collective dynamics is described by a system of six equations (three for the variables of the average $X=\left[\left\langle v_{1}\right\rangle,\left\langle v_{2}\right\rangle,\langle I\rangle\right]$ and three for the shape parameter $\left.W\right)$. This can be compactly written as Eq. (6), where $f(X)$ is a vector of components:

$$
\begin{gathered}
f(1)=-\frac{G^{b}}{C^{1}} v^{1}+\frac{v^{2}-v^{1}}{R C^{1}}-\frac{G^{a}-G^{b}}{2 C^{1}}\left(\left|v^{1}+B^{p}\right|-\left|v^{1}-B^{p}\right|\right), \\
f(2)=\frac{1}{C^{2}}\left(I+\frac{v^{1}-v^{2}}{R}\right),
\end{gathered}
$$

$$
f(3)=-\frac{1}{L}\left(I r^{b}-v^{2}\right) .
$$

The coupling matrix $\mathcal{K}$ is

$$
\mathcal{K}=\left[\begin{array}{ccc}
\frac{2}{C^{1} R_{1}^{k}} & 0 & 0 \\
0 & \frac{2}{C^{2} R_{2}^{k}} & 0 \\
0 & 0 & 0
\end{array}\right]
$$

and the Jacobian matrix $\mathcal{J}$ is

$$
\mathcal{J}=\left[\begin{array}{ccc}
j_{1,1} & \frac{1}{C^{1} R} & 0 \\
\frac{1}{C^{2} R} & -\frac{1}{C^{2} R} & \frac{1}{C^{2}} \\
0 & -\frac{1}{L} & -\frac{r^{b}}{L}
\end{array}\right],
$$

where

$$
\begin{aligned}
j_{1,1}= & -\frac{G^{b}}{C^{1}}+\frac{1}{R C^{1}}+\frac{G^{a}-G^{b}}{2 C^{1}} \\
& \times\left[\operatorname{sgn}\left(v^{1}+B^{p}\right)-\operatorname{sgn}\left(v^{1}-B^{p}\right)\right] .
\end{aligned}
$$

[1] Synchronization: Theory and Application, edited by A. Pikovsky and Y. Maistrenko (Kluwer, Dordrecht, 2003).

[2] E. Mosekilde, Y. Maistrenko, and D. Postnov, Chaotic Synchronization: Applications to Living Systems (World Scientific, Singapore, 2002).

[3] S. C. Manrubia, A. S. Mikhailov, and D. H. Zanette, Emergence of Dynamical Order: Synchronization Phenomena in Complex Systems (World Scientific, Singapore, 2004).

[4] R. A. Oliva and S. H. Strogatz, Int. J. Bifurcation Chaos Appl. Sci. Eng. 11, 2359 (2001).

[5] Y. Kuramoto, Chemical Oscillations, Waves and Turbulence (Springer, Berlin, 1984).

[6] I. Z. Kiss, Y. Zhai, and J. L. Hudson, Science 296, 1676 (2002).

[7] A. T. Winfree, The Geometry of Biological Time, 2nd ed. (Springer, New York, 2001).

[8] S. Danø, F. Hynne, S. D. Monte, F. d'Ovidio, P. G. Sørensen, and H. Westerhoff, Faraday Discuss. 120, 261 (2001).

[9] G. V. Osipov and M. M. Sushik, Phys. Rev. E 58, 7198
(1998).

[10] P. C. Matthews, R. E. Mirollo, and S. H. Strogatz, Physica D 52, 293 (1991).

[11] M. G. Rosenblum, A. S. Pikovsky, and J. Kurths, Phys. Rev. Lett. 76, 1804 (1996).

[12] Y. Kuramoto, in International Symposium on Mathematical Problems in Theoretical Physics, edited by H. Araki, Vol. 39 of Lecture Notes in Physics (Springer, New York, 1975), p. 420.

[13] I. Z. Kiss, Y. Zhai, and J. L. Hudson, Science 296, 1676 (2002).

[14] S. De Monte and F. d'Ovidio, Europhys. Lett. 58, 21 (2002).

[15] S. De Monte, F. d'Ovidio, and E. Mosekilde, Phys. Rev. Lett. 90, 054102 (2003).

[16] S. De Monte, F. d'Ovidio, H. Chaté, and E. Mosekilde, Phys. Rev. Lett. 92, 254101 (2004).

[17] G. B. Ermentrout and W. C. Troy, SIAM J. Math. Anal. 20, 1436 (1989).

[18] G. B. Ermentrout, Physica D 41, 219 (1990).

[19] F. M. Atay, Phys. Rev. Lett. 91, 094101 (2003). 